\title{
Programa de Alimentação do Trabalhador: avaliando o conhecimento por parte dos gestores administrativos e técnicos
}

Worker's Food Program: evaluating the knowledge of administrative and technical managers

Lúcia Chaise Bories'

Janaína Santos de Lima'

' Curso de Nutrição. Universidade Comunitária da Região de Chapecó. Chapecó, SC, Brasil.

Fonte financiadora: FAPESC - Chamada Pública 11/2009 - Prêmio Mérito Universitário Catarinense.

Correspondência / Correspondence Lúcia Chaise Borjes

E-mail: Ibories@unochapeco.edu.br

\section{Resumo}

O Programa de Alimentação do Trabalhador (PAT) tem por objetivo melhorar as condições nutricionais dos trabalhadores. Este estudo buscou avaliar o conhecimento dos gestores administrativos e técnicos do PAT sobre promoção de alimentação saudável e saúde, e a interface deste tema com o programa. O estudo foi composto por oito empresas cadastradas no PAT, localizadas em Chapecó-SC, com mais de 150 funcionários, que possuíam refeitório e contavam com nutricionista. Aplicou-se um questionário, e as gravações foram transcritas e analisadas por meio da técnica de análise do conteúdo. Sobre o motivo de a empresa ter sido cadastrada no PAT, entre as expressões-chave, os gestores administrativos referem-se ao benefício fiscal concedido à empresa. Já os gestores técnicos com maior frequência enfatizaram os benefícios para o trabalhador. Perguntados sobre o que é o PAT, os gestores administrativos comentaram com maior frequência a respeito do bem-estar para o funcionário. Sobre a mesma questão, os gestores técnicos citam mais características sobre o programa. Verificou-se que grande parte dos gestores técnicos e administrativos responsáveis pelo PAT apresenta conhecimento vago sobre o programa. Nas falas, observou-se que muitos gestores deixam mais explícitos os objetivos fiscais para a empresa, não ressaltando os objetivos principais relativos ao trabalhador; no entanto, os gestores técnicos demonstram maior esclarecimento sobre o papel do PAT com a saúde do trabalhador do que os gestores administrativos.

Palavras-chave: Trabalhadores. Alimentação Coletiva. Programas e Políticas de Nutrição e Alimentação. Avaliação de Programas e Projetos de Saúde. 


\section{Abstract}

The Workers' Food Program (WFP) aims to improve the nutritional status of workers. This study evaluated the knowledge of WFP's administrative and technical managers promoting healthy diet and health, and the interface of this issue with the program. The study comprised eight companies registered in WFP, located in the city of Chapeco-SC, Brazil, with over 150 employees, who had dining hall and nutritionists. We applied a questionnaire, and the recordings were transcribed and analyzed using the technique of content analysis. On why the company has been registered in WFP, between the key expressions, administrative managers refer to the tax benefit granted to the company. Technical managers most frequently emphasized the benefit to the workers. Asked what WFP is, the administrative managers commented most frequently about the employee's welfare. On the same subject, the technical managers cited more features about the program. It was found that most of the technical and administrative managers responsible for WFP had vague knowledge about the program. In the speech, it was observed that many managers leave more explicit fiscal objectives for the company, not highlighting the main objectives for employees; however, the technical managers demonstrated greater clarity on the role of WFP with the health worker than administrative managers.

Key words: Workers. Collective Feeding. Nutrition Programs and Policies. Program Evaluation.

\section{Introdução}

O Programa de Alimentação do Trabalhador (PAT) foi instituído pela Lei nº 6.321, de 14 de abril de 1976, e regulamentado pelo Decreto n'. 5, de 14 de janeiro de 1991. Com parceria entre governo, empresa e trabalhador, o PAT prioriza o atendimento aos trabalhadores de baixa renda (inferior a cinco salários mínimos mensais), tendo por objetivo melhorar as condições nutricionais dos trabalhadores, com repercussões positivas na qualidade de vida, redução de acidentes de trabalho e aumento da produtividade. ${ }^{1}$

Dados do Ministério do Trabalho e Emprego demonstram que, em 2007, 10.057.848 de trabalhadores foram beneficiados com o programa no país, sendo 464.741 em Santa Catarina; destes, 285.664 recebem refeições na própria empresa e 91.784 recebem refeição por meio de convênio. 
Na refeição por meio de convênio, os trabalhadores da empresa beneficiária fazem suas refeições em restaurantes conveniados com empresas operadoras de vales, tíquetes, cupons, cheques, etc. ${ }^{2}$

Geraldo, Bandoni \& Jaime ${ }^{3}$ avaliaram os aspectos dietéticos da alimentação oferecida por empresas participantes do PAT e os resultados mostraram a ocorrência de elevados percentuais de inadequação para todas as variáveis estudadas em relação às metas de ingestão de nutrientes recomendadas pela OMS para a prevenção de doenças crônicas e a promoção de alimentação saudável.

Como consequência do atual cenário epidemiológico da população adulta brasileira, em 2006 os parâmetros nutricionais do PAT foram alterados. Anteriormente, as refeições principais deveriam ter no mínimo $1.400 \mathrm{kcal}$ e as refeições menores no mínimo $300 \mathrm{kcal}$. As novas recomendações preconizam de 600 a $800 \mathrm{kcal}$ nas refeições maiores, e as refeições menores de 300 a $400 \mathrm{kcal}$, admitindo-se acréscimo de $20 \%$ para ambas em relação ao valor energético total. ${ }^{4}$

Além disso, a partir da década de 1990, foi incluída no programa a promoção da alimentação saudável, estimulando as empresas a realizarem ações de educação nutricional. ${ }^{5}$ Em 1999, por meio da Comissão Tripartite, formada por representantes do governo, trabalhadores e empregadores, foi publicada a cartilha Orientação da Educação Alimentar, com o objetivo de direcionar ações de educação alimentar. ${ }^{6}$ Essa comissão, em conjunto com a Coordenação do PAT, propõe ações para a implementação de educação alimentar e nutricional dos trabalhadores, visando melhorar seu estado nutricional e reduzir a incidência de doenças crônicas não transmissíveis. ${ }^{7}$

O ambiente de trabalho é reconhecido como um local estratégico de promoção da saúde e alimentação saudável. ${ }^{8}$ A Organização Mundial da Saúde considera que o local de trabalho deve dar a oportunidade e estimular os trabalhadores a fazerem escolhas saudáveis. ${ }^{9}$ Nesse sentido, a consolidação do PAT como um programa que visa à promoção da alimentação saudável no ambiente de trabalho demanda que seus gestores diretos, responsáveis por sua execução nas empresas, compreendam e aceitam essa questão.

Com base no exposto, foi realizado estudo nas empresas cadastradas no PAT no município de Chapecó, estado de Santa Catarina, com o intuito de avaliar o conhecimento dos gestores administrativos e técnicos do PAT sobre promoção de alimentação saudável e saúde e a interface do tema com o programa.

\section{Metodologia}

Trata-se de estudo de caso com caráter descritivo e abordagem quantitativa, no qual a população do estudo foi composta por empresas cadastradas no PAT, de acordo com a Relação de Empresas Beneficiárias, disponível no site do MTE, baseada nos relatórios a partir de $2008 .{ }^{2}$ Nos critérios de 
inclusão iniciais, as empresas deveriam estar localizadas no município de Chapecó-SC e possuir mais de 150 funcionários. A seleção da amostra realizou-se no mês de março de 2010, quando foram identificadas 12 empresas ( $\mathrm{N}=$ tamanho da população). Após essa primeira seleção, foram ainda excluídas as empresas que forneciam cesta básica ou vale-refeição, que não possuíssem refeitório ou que não contassem com nutricionista responsável. Deste modo, finalizou-se uma amostra (n) de oito empresas.

Com o objetivo de determinar o conhecimento sobre o PAT, foi aplicado um questionário com questões discursivas contendo quatro perguntas: 1) Na sua opinião, por que essa empresa foi cadastrada no PAT?; 2) Se você tivesse que explicar para alguém o que é PAT, o que você falaria?; 3) Você conhece a cartilha Orientação da Educação Alimentar do TEM, de 1999? Se a resposta fosse afirmativa, seguiria para a última pergunta; 4) Você faz alguma ação voltada para Orientação da Educação Alimentar, conforme proposto na cartilha?

O questionário foi aplicado aos gestores administrativos (independentemente da sua formação: proprietários, administradores, entre outros - 8 indivíduos) e aos técnicos (nutricionistas - 8 indivíduos) responsáveis pelo programa na empresa, totalizando 16 entrevistados. Essa etapa ocorreu entre abril e maio de 2010, e as entrevistas foram gravadas, mediante aceite de participação, sendo que todos assinaram o Termo de Consentimento Livre e Esclarecido (TCLE).

As gravações foram transcritas e analisadas por meio da técnica de análise do conteúdo, permitindo, neste estudo, a identificação dos aspectos pela frequência. Análise de conteúdo é um conjunto de técnicas de análise da comunicação. Esta técnica pode ser quantitativa e qualitativa. Na primeira se descreve uma frequência das características que se repetem no conteúdo do texto. E, na segunda, se observa a presença ou não de determinada característica ou conjunto de características de conteúdo em determinada fração do conteúdo. ${ }^{10}$

Após a transcrição, procedeu-se à organização das informações através da categorização das respostas. ${ }^{11}$ Essa etapa consistiu basicamente em analisar o material verbal, transcrito das entrevistas, buscando as expressões-chave. A categorização não levou em consideração somente o número de entrevistados, mas considerou o número de vezes que a resposta apareceu, porque alguns entrevistados deram mais de uma resposta para a mesma pergunta. Assim, a opção de corte foi frequencial.

\section{Resultados}

Das respostas dos profissionais foram extraídas expressões-chave, e estas foram agrupadas em ideias centrais. O quadro 1 apresenta a categorização das respostas. Embora o n tenha sido 8, em algumas perguntas aparecem respostas em número superior, pois o mesmo entrevistado deu 
mais de uma resposta para a mesma pergunta. Por esse motivo, o n de cada pergunta pode ser maior que o n total da amostra.

Quando indagados sobre o porquê de a empresa ter sido cadastrada no PAT, entre as expressões-chave, os gestores administrativos referem-se ao benefício fiscal concedido à empresa $(n=5)$. Segundo um dos profissionais, a empresa foi cadastrada "pra se valer dos benefícios que o Programa de Alimentação do Trabalhador oferece à empresa cadastrada no PAT”. Esses profissionais citam o benefício oferecido para o trabalhador $(n=5)$, e também que acreditam que a empresa foi cadastrada em razão do cumprimento da legislação $(n=1)$.

Em relação aos gestores técnicos no presente estudo, percebeu-se que, apesar de também citarem o benefício fiscal à empresa $(n=3)$, com maior frequência enfatizaram os benefícios para o trabalhador $(n=6)$ como um dos motivos que levaram a empresa a se cadastrar no programa. As demais opiniões dos gestores técnicos são de que o motivo foi a exigência da empresa contratante $(n=1)$ no caso das empresas terceirizadas, e melhoria da produção $(n=1)$.

Em nenhum momento os gestores administrativos, nem os gestores técnicos, citaram os benefícios para o governo, entre eles: redução de despesas e investimentos na área da saúde, crescimento da atividade econômica e bem-estar social.

Perguntados sobre o que é o PAT, os gestores administrativos comentaram com maior frequência a respeito do bem-estar para o funcionário $(n=4)$. Seguido, em termos de frequência, que é um benefício concedido para empresa $(n=3)$ e é o programa de alimentação do trabalhador $(n=3)$. Foi comentado que é um programa do governo federal $(n=1)$, que auxilia na melhoria da nutrição dos trabalhadores com renda até cinco salários mínimos $(n=1)$. Percebe-se que os gestores administrativos apresentam conhecimento superficial a respeito do programa, focando em alguns aspectos referentes aos benefícios oferecidos para a empresa e para o trabalhador, mas seu conhecimento é vago.

Os gestores técnicos, quando comparados aos administrativos, perguntados sobre o que é o PAT, citaram mais características sobre o programa. Conforme a fala de um dos profissionais, o PAT

[...] é o programa de alimentação do trabalhador, que onde a empresa se preocupa em fornece uma alimentação equilibrada em quantidade e qualidade aos funcionários, além de todo o trabalho com...ãhm... com esse acompanhamento com a nutricionista [...].

Os profissionais comentaram com maior frequência sobre a alimentação equilibrada fornecida ao trabalhador $(n=5)$ e também que é o programa de alimentação do trabalhador $(n=4)$, visa ao benefício para a empresa, como a produtividade $(n=4)$. Em menor frequência, foi citada a educação nutricional $(n=1)$, programa do governo federal $(n=1)$, que o programa mantém o funcionário na empresa $(n=1)$ e um profissional disse não saber responder. 
Quando perguntados sobre a cartilha da educação alimentar do Ministério do Trabalho e Emprego (MTE), verificou-e que os gestores administrativos desconhecem-na $(n=4)$ ou não sabem explicá-la corretamente $(n=4)$. Em relação aos gestores técnicos, apenas um profissional afirmou e demonstrou conhecer a cartilha; os demais, assim como os profissionais da área administrativa, não conhecem a cartilha $(n=5)$ ou não sabem explicá-la corretamente $(n=2)$.

Aos gestores administrativos e técnicos que afirmaram conhecer a cartilha, foi questionado se realizavam ações voltadas para a orientação da educação alimentar conforme proposto nela. Entre os gestores administrativos, um airmou não realizar; os demais ( $\mathrm{n}=3)$ afirmaram que quem faz as ações é a nutricionista. Dentre os gestores técnicos, quatro profissionais comentaram que realizam educação nutricional, seja através de murais ou por meio dos estagiários.

Quadro 1. Categorização das respostas. Chapecó-SC, 2010.

\begin{tabular}{|l|l}
\hline Expressões-chave & Ideia central
\end{tabular}

1. Na sua opinião, por que essa empresa foi cadastrada no PAT?

Benefício para o trabalhador

Melhor qualidade de vida para o trabalhador Benefício para o trabalhador

Oferecer alimentação ao funcionário

Benefício fiscal para empresa Benefício fiscal para empresa

Cumprimento da legislação Cumprimento da legislação

Exigência da contratante Exigência da contratante

Melhoria da produção Melhoria da produção

\section{Se você tivesse que explicar para alguém o que é o PAT, o que você falaria?}

Bem estar para o funcionário

Benefício para o trabalhador

Garantir a saúde do trabalhador

Bem estar para o funcionário

Alimentação equilibrada 


\begin{tabular}{|c|c|}
\hline Expressões-chave & Ideia central \\
\hline \multicolumn{2}{|l|}{ É o Programa de Alimentação do } \\
\hline Trabalhador & É o Programa de Alimentação do \\
\hline É o Programa de Alimentação ao & Trabalhador \\
\hline \multicolumn{2}{|l|}{ Trabalhador } \\
\hline \multicolumn{2}{|l|}{ Benefício para a empresa } \\
\hline \multicolumn{2}{|l|}{ alimentação } \\
\hline Programa do Governo Federal & Programa do Governo Federal \\
\hline Auxilia na melhoria da nutrição dos & Auxilia na melhoria da nutrição dos \\
\hline trabalhadores com renda até 5 SM & trabalhadores com renda até $5 \mathrm{SM}$ \\
\hline Educação nutricional & Educação nutricional \\
\hline Manter o funcionário na empresa & Manter o funcionário na empresa \\
\hline Não sabe & Não sabe \\
\hline \multicolumn{2}{|c|}{ 3. Você conhece a cartilha Orientação da Educação Alimentar do MTE de 1999? } \\
\hline Não & Não \\
\hline Não soube explicar corretamente & Não soube explicar corretamente \\
\hline Sim & Sim \\
\hline \multicolumn{2}{|c|}{$\begin{array}{l}\text { 4. Você faz alguma ação voltada para Orientação da Educação Alimentar conforme } \\
\text { proposto na cartilha? }\end{array}$} \\
\hline Não responderam & Não responderam \\
\hline $\begin{array}{l}\text { A nutricionista faz (mas não soube explicar } \\
\text { exatamente o que é cartilha) }\end{array}$ & $\begin{array}{l}\text { A nutricionista faz (mas não soube } \\
\text { explicar exatamente o que é cartilha) }\end{array}$ \\
\hline Não & Não \\
\hline \multicolumn{2}{|l|}{ Educação nutricional através de murais } \\
\hline Educação nutricional & Educação nutricional \\
\hline Educação nutricional por estagiários & \\
\hline
\end{tabular}

Fonte: elaboração própria. 


\section{Discussão}

Assim como no presente estudo, Bandoni, Brasil \& Jaime ${ }^{12}$ analisaram o conhecimento dos gestores do PAT e verificaram que 32,2\% das expressões-chave enfatizavam os benefícios do programa para a empresa. Ainda em relação à inscrição das empresas no programa, os autores citam em seu estudo que a ideia central "por causa da lei" revela uma visão equivocada da natureza do programa, cuja inscrição é voluntária por parte das empresas interessadas. ${ }^{12}$

Para a empresa, além do incentivo fiscal (dedução de até 4\% no imposto de renda devido), o programa oferece outros benefícios, como: aumento de produtividade, maior integração entre trabalhador e empresa, redução do absenteísmo (atrasos e faltas), redução da rotatividade e isenção de encargos sociais sobre o valor da alimentação fornecida. ${ }^{7}$

Deve-se destacar que o objetivo principal do PAT não é a isenção fiscal, mas a melhoria da situação nutricional dos trabalhadores, visando a promoção da saúde e prevenção de doenças relacionadas ao trabalho. ${ }^{7}$ Segundo Veloso \& Santana, ${ }^{13}$ no Brasil as políticas de alimentação para o trabalhador se fundamentam na ideia de que a força de trabalho é elemento-chave para a produção econômica.

Uma alimentação balanceada é fundamental no que diz respeito a saúde e produtividade do trabalhador. Estudos científicos confirmam a combinação entre uma alimentação equilibrada e a produtividade, assim como o baixo rendimento do trabalho quando ocorre ingestão calórica inadequada. ${ }^{14}$ Isto evidencia a importância da alimentação no equilíbrio orgânico, não apenas como fator para a manutenção da saúde, mas, do ponto de vista econômico, como condição para maior dispêndio de energia e, consequentemente, maior capacidade de trabalho. ${ }^{15}$

Sabe-se que a má nutrição pode desencadear consequências relacionadas à redução da vida média, produtividade, resistência às doenças, aumento à predisposição aos acidentes de trabalho e baixa capacidade de aprendizado do trabalho. Ressalta-se, ainda, que a oferta de refeições aos trabalhadores durante a jornada de trabalho pode representar um acréscimo de $10 \%$ na produção. ${ }^{14,16}$

A alimentação e a nutrição constituem requisitos básicos para a promoção e a proteção da saúde, possibilitando a afirmação plena do potencial de crescimento e desenvolvimento humano, com qualidade de vida e cidadania. ${ }^{17}$ A alimentação pouco saudável e a falta de atividade física são as principais causas das doenças não transmissíveis mais importantes, como as cardiovasculares, a diabetes tipo 2 e determinados tipos de câncer, e contribuem substancialmente para a carga mundial de morbidade, mortalidade e incapacidade. ${ }^{18}$

Savio et al. ${ }^{19}$, em seu estudo, ressaltam a importância e a necessidade do nutricionista à frente da Unidade de Alimentação e Nutrição (UAN), como agente promotor da saúde para a vasta clientela frequentadora desse mercado em expansão. Conforme a Resolução CFN nº 380/2005, 
compete ao nutricionista, no exercício de suas atribuições na alimentação do trabalhador, além de planejar, organizar, dirigir, supervisionar, avaliar os serviços de alimentação e nutrição do PAT, realizar e promover a educação nutricional e alimentar ao trabalhador em instituições públicas e privadas, por meio de ações, programas e eventos, visando a prevenção de doenças e promoção e manutenção de saúde. ${ }^{20}$

De acordo com Bandoni, Brasil \& Jaime ${ }^{12}$, é preocupante a constatação do desconhecimento sobre o PAT pelos responsáveis diretos, que em seu estudo representou 27,7\% das expressões-chave identificadas, não o reconhecendo como um programa social. De acordo com os autores, como a participação no programa não exige capacitação ou qualificação dos executores, sendo apenas necessário o preenchimento de formulários habilitando a empresa a participar, essas respostas podem ser parcialmente justificadas. Desta forma, o aumento de informação sobre o programa e a capacitação dos gestores locais são ações fundamentais na efetivação do potencial de promoção de alimentação saudável que o PAT apresenta.

A cartilha da educação alimentar do MTE, apesar de ser de 1999, é uma ferramenta importante, pois aborda a educação alimentar, sua importância, os benefícios para a empresa, para o trabalhador e para a sociedade. Além disso, aponta estratégias simples para se realizar educação nutricional no ambiente de trabalho, dicas conforme o tipo de serviço prestado e em anexo contém um questionário para diagnóstico de saúde, exemplos de cardápios e dicas para as doenças crônicas não transmissíveis. ${ }^{6}$

Percebe-se que o estímulo ao desenvolvimento por parte das empresas de ações educativas de promoção à saúde é um objetivo a ser perseguido. ${ }^{21} \mathrm{~A}$ inserção da educação alimentar reflete uma tentativa de deslocar a alimentação do trabalhador do foco genuinamente energético para um enfoque de promoção à saúde e/ou prevenção de enfermidades. ${ }^{22}$ No entanto, apesar da obrigatoriedade da empresa em seguir as exigências nutricionais e proporcionar educação alimentar e nutricional aos trabalhadores beneficiados, um dos pontos fracos do programa é justamente a carência de programas e atividades de educação alimentar, visando promover a saúde e incentivar as boas práticas alimentares e de vida saudável. ${ }^{7}$

Cabe ao nutricionista preocupar-se com a prevenção de doenças e a promoção da saúde. Além de bem nutrir, uma alimentação equilibrada poderá servir de exemplo para a criação de hábitos alimentares adequados nos comensais. Sendo assim, é de extrema importância a adequação das refeições servidas numa UAN, e estratégias de promoção da saúde a fim de melhorar a qualidade de vida devem ser incentivadas. ${ }^{14}$

Sabe-se, entretanto, que os conhecimentos básicos da nutrição e sua eficaz implementação ficam, no cotidiano dos nutricionistas de UANs, secundários em detrimento ao controle de custos, exigência que é supervalorizada pelos empresários. $\mathrm{O}$ alimento em si fica sem sentido se o 
nutricionista não fizer a associação do alimento, do ato de alimentar-se e do indivíduo. Para isso, a formação do nutricionista deve ser orientada para que se ensine e se aprenda ao mesmo tempo, numa construção e produção do conhecimento em alimentação e nutrição. ${ }^{23}$

O nutricionista, como educador, deve ter a certeza de que ensinar não é transferir conhecimento, mas criar possibilidades para sua construção. A educação nutricional se dá durante todo o processo, desde a produção do alimento até a hora da sua distribuição como uma orientação constante, não só em nível de informação, mas, principalmente, de conscientização. ${ }^{24}$

A formação do nutricionista como vem sendo feita atualmente, buscando um profissional com desempenho técnico específico, não pode mais ser aceita. As instituições de ensino superior, além de formar cidadãos com competência técnica e científica, precisam capacitar os futuros profissionais para compreender a realidade. ${ }^{25}$ "A maior ênfase ao conteúdo biológico, em detrimento do social, dificulta ao acadêmico o aprofundamento de conhecimentos que lhe permitiriam ter melhor compreensão dos determinantes dos problemas nutricionais". ${ }^{26}$ A falta de articulação entre a teoria e a prática durante a graduação acaba formado futuros profissionais deficientes. ${ }^{27}$

O desafio de uma alimentação saudável implica a mobilização do poder público e do segmento da produção, transformação e comercialização de alimentos dentro de uma nova ética que considere a saúde como fator essencial e orientador das estratégias e relações sociais e econômicas intra e interpaíses. ${ }^{28}$

O governo brasileiro, com o intuito de suplementar a renda da parcela da população que não alcança o nível de renda mínimo, utiliza programas de ação como forma indireta de distribuição de renda e incremento ao crescimento e desenvolvimento econômico. ${ }^{15}$ Porém, conforme Santos et $a l .^{22}$, faz-se necessário que a avaliação de programas constitua uma das atividades básicas e contínuas por parte do governo. Isso permitiria a reorientação e/ou reformulação de suas estratégias, para que se alcancem os objetivos almejados.

Precisa-se avaliar a eficiência dos programas, não apenas em relação ao resultado obtido e à quantidade de recursos alocados, mas considerando as dificuldades ou potencialidades existentes na região em que os programas estão funcionando. ${ }^{29}$

O Sistema Único de Saúde (SUS), em seu programa Humaniza SUS, mostra que "um dos aspectos que mais tem chamado a atenção quando da avaliação dos serviços é o despreparo dos profissionais para lidar com a dimensão subjetiva que toda prática de saúde supõe $" .{ }^{30}$ A educação em saúde contempla a educação nutricional e as práticas de educação em saúde deveriam estar inseridas nas políticas de saúde do país e serem efetivas. 


\section{Conclusão}

Verificou-se que grande parte dos gestores técnicos e administrativos responsáveis pelo PAT apresenta conhecimento vago sobre o programa. Nas falas, observou-se que muitos gestores deixam mais explícitos os objetivos fiscais para a empresa, não ressaltando os objetivos principais que são referentes ao trabalhador. No entanto, os gestores técnicos demonstram maior conscientização do papel do PAT para com a saúde do trabalhador do que os gestores administrativos.

Levando em consideração os resultados do estudo, também se faz necessária maior divulgação de informações sobre o PAT junto à população-alvo, de forma a conscientizar os trabalhadores sobre seus direitos e ações desenvolvidas pelo programa, informar e conscientizar também os gestores administrativos e técnicos, envolvidos diretamente com o PAT e que apresentam responsabilidades a cumprir.

\section{Referências}

1. Brasil. Ministério do Trabalho e Emprego. Decreto $n^{\circ}$ 5, Regulamenta a Lei $n^{\circ}$ 6.321, de 14 de abril de 1976, que trata do Programa de Alimentação do Trabalhador. 1991 jan14. Diário Oficial da União. 14 jan. 1991; 1(1058).

2. Brasil. Ministério do Trabalho e Emprego. Relatórios e gráficos do PAT a partir de 2008. Brasília: Ministério do Trabalho e Emprego; 2008. Disponível em: http://www.mte.gov.br/pat/default.asp.

3. Geraldo APG, Bandoni DH, Jaime PC. Aspectos dietéticos das refeições oferecidas por empresas participantes do Programa de Alimentação do Trabalhador na Cidade de São Paulo, Brasil. Rev. Panam. Salud Publica. 2008; 23(1):19-25.

4. Brasil. Ministério do Trabalho e Emprego. Portaria n 193, de 05 dez. 2006. Altera os parâmetros nutricionais do Programa de Alimentação do Trabalhador - PAT. Diário Oficial da União. 07 dez. 2006.

5. Jaime PC, Bandoni DH, Geraldo APG, Rocha RV. Adequação das refeições oferecidas por empresas cadastradas no Programa de Alimentação do Trabalhador na Cidade de São Paulo. O Mundo da Saúde. 2005; 29(2):186-191.

6. Brasil. Ministério do Trabalho e Emprego. Orientação da educação alimentar. Brasília: Ministério do Trabalho e Emprego; 1999. Disponível em: http://www.mte.gov.br/pat/oea.pdf.

7. Brasil. Ministério do Trabalho e Emprego. Programa de Alimentação do Trabalhador. Brasília: Ministério do Trabalho e Emprego; 2004. Disponível em: http://www.mte.gov.br/Empregador/pat/

8. Chu C, Breucker G, Harris N, Stitzel A, Gan X, Gu X, Dwyer S. Health promoting workplaces: international settings development. Health Promot. Int. 2000; 15(2):157-167. 
9. World Health Organization. Global strategy on diet, physical activity and health, 2004. Food Nutr Bull. 2004; 25(3):292-302.

10. Rocha D, Deusdara B. Análise de conteúdo e análise do discurso: aproximações e afastamentos na (re)construção de uma trajetória. Alea. 2005; 7(2):305-322.

11. Lima MADS. Análise de conteúdo: estudo e aplicação. Rev. Logos. 1993; (1):53-8.

12. Bandoni DH, Brasil BG, Jaime PC. Programa de Alimentação do Trabalhador: representações sociais de gestores locais. Rev. Saúde Pública. 2006; 40(5):837-842.

13. Veloso IS, Santana VS. Impacto nutricional do programa de alimentação do trabalhador no Brasil. Rev. Panam. Salud Publica. 2002; 11(1):24-31.

14. Vanin M, Southier N, Novello D, Francischetti VA. Adequação nutricional do almoço de uma unidade de alimentação e nutrição de Guarapuava, PR. Rev. Salus. 2007; 1(1):31-38.

15. Moura JB. Avaliação do programa de alimentação do trabalhador, no Estado de Pernambuco, Brasil. Rev. Saúde Pública. 1986; 20(2):115-128.

16. Bandoni DH, Jaime PC. A qualidade das refeições de empresas cadastradas no Programa de Alimentação do Trabalhador na cidade de São Paulo. Rev. Nutr. 2008; 21(2):177-184.

17. Brasil. Ministério da Saúde. Política Nacional de Alimentação e Nutrição. Brasília: Ministério da Saúde; 2003. Disponível em: http://189.28.128.100/nutricao/docs/geral/pnan2011.pdf.

18. Organização Mundial da Saúde. Estratégia Global em Alimentação Saudável, Atividade Física e Saúde. $57^{\text {a }}$ Assembléia Mundial de Saúde; 22 maio 2004. Disponível em: http://www.prosaude.org/ publica coes/diversos/Estrategia_Global_portugues.pdf.

19. Savio KEO, Costa THM, Miazaki E, Schmitz BAS. Avaliação do almoço servido a participantes do programa de alimentação do trabalhador. Rev. Saúde Pública. 2005; 39(2):148-155.

20. Brasil. Conselho Federal de Nutricionistas. Resolução CFN n 380, 09 dez. 2005. Dispõe sobre a definição das áreas de atuação do nutricionista e suas atribuições, estabelece parâmetros numéricos de referência por área de atuação e dá outras providências. Diário Oficial da União. 10 jan. 2005; Seção 1.

21. Burlandy L, Anjos LA. Acesso a vale-refeição e estado nutricional de adultos beneficiários do Programa de Alimentação do Trabalhador no Nordeste e Sudeste do Brasil, 1997. Cad. Saúde Pública. 2001; 17(6):1457-1464.

22. Santos LMP, Santos SMC, Santana LAA, Henrique FCS, Mazza, RPD, Santos LAS, Santos LS. Avaliação de políticas públicas de segurança alimentar e combate à fome no período 1995-2002: 4 Programa Nacional de Alimentação Escolar. Cad. Saúde Pública. 2007; 23(11):2681-2693.

23. Sousa AA, Proença RPC. Tecnologias de gestão dos cuidados nutricionais: recomendações para qualificação do atendimento nas unidades de alimentação e nutrição hospitalares. Rev. Nutr. 2004; 17(4):425-436. 
24. Manço AM, Costa FNA. Educação nutricional: caminhos possíveis. Alim. Nutr. 2004; 15(2):145-153.

25. Amorim STSP, Moreira H, Carraro TE. A formação de pediatras e nutricionistas: a dimensão humana. Rev. Nutr. 2001; 14(2):111-118.

26. Bosi MLM. A face oculta da nutrição: ciência e ideologia. Rio de Janeiro: Espaço e Tempo; 1988. 220p.

27. Fávero ML. A Universidade e estágio curricular: subsídios para discussão. In: Alves N, organizador. Formação de professores: pensar e fazer. 2. ed. São Paulo: Cortez; 1993. 103 p.

28. Barreto SM, Pinheiro ARO, Sichieri R, Monteiro CA, Batista Filho M, Schimidt MI, Lotufo P, Assis AM, Guimarães V, Recine EGIG, Victora CG, Coitinho D, Passos VMA. Análise da estratégia global para alimentação, atividade física e saúde, da Organização Mundial da Saúde. Epidemiol. Serv. Saúde. 2005; 14(1):41-68.

29. Jannuzzi PM. Indicadores para diagnóstico, monitoramento e avaliação de programas sociais no Brasil. Rev. Serviço Público. 2005; 56(2):137-160.

30. Brasil. Ministério da Saúde. Humaniza-SUS: Política Nacional de Humanização. Brasília: Ministério da Saúde; 2004.

Recebido: 29/6/2013

Revisado: 04/10/2013

Aprovado: 07/1/2014 
